\title{
Experiments and modeling of ballistic penetration using an energy failure criterion
}

\author{
M. Dolinski and D. Rittel ${ }^{\mathrm{a}}$ \\ Faculty of Mechanical Engineering, Technion - Israel Institute of Technology, Israel
}

\begin{abstract}
One of the most intricate problems in terminal ballistics is the physics underlying penetration and perforation. Several penetration modes are well identified, such as petalling, plugging, spall failure and fragmentation (Sedgwick, 1968). In most cases, the final target failure will combine those modes. Some of the failure modes can be due to brittle material behavior, but penetration of ductile targets by blunt projectiles, involving plugging in particular, is caused by excessive localized plasticity, with emphasis on adiabatic shear banding (ASB). Among the theories regarding the onset of ASB, new evidence was recently brought by Rittel et al. (2006), according to whom shear bands initiate as a result of dynamic recrystallization (DRX), a local softening mechanism driven by the stored energy of cold work. As such, ASB formation results from microstructural transformations, rather than from thermal softening. In our previous work (Dolinski et al., 2010), a failure criterion based on plastic strain energy density was presented and applied to model four different classical examples of dynamic failure involving ASB formation. According to this criterion, a material point starts to fail when the total plastic strain energy density reaches a critical value. Thereafter, the strength of the element decreases gradually to zero to mimic the actual material mechanical behavior.

The goal of this paper is to present a new combined experimental-numerical study of ballistic penetration and perforation, using the above-mentioned failure criterion. Careful experiments are carried out using a single combination of AISI 4340 FSP projectiles and $25[\mathrm{~mm}]$ thick RHA steel plates, while the impact velocity, and hence the imparted damage, are systematically varied. We show that our failure model, which includes only one adjustable parameter in this present work, can faithfully reproduce each of the experiments without any further adjustment.

Moreover, it is shown that the most common failure criterion based on a critical strain is simply inadequate to reproduce the results, due to the linear nature of the damage evolution. The advantages of the energy-based failure criterion are discussed in detail.
\end{abstract}

\section{Introduction}

One of failure modes in high strain rates applications consists of localization of the plastic deformation into a narrow band or surface. This failure mode is referred to as adiabatic shear banding (ASB), and was first documented by Tresca (1879), who observed it while forging platinum billets. ASB occurs in various applications such as machining, high velocity plastic processing, and plays a major role in the context of terminal ballistics. ASB's are frequently encountered in materials that have thermal softening and quite low strain hardening. Zener and Hollomon (1944) suggested that ASB initiates when the adiabatic thermal softening exceeds the strain hardening. Consequently, the traditional way to model ASB formation in a dynamically deformed solid consists of introducing a critical failure strain, the latter being calculated based on the competing mechanisms of strain hardening and thermal softening.

In the recent years, Rittel et al. (2006) challenged this assumption and proposed that the dynamic stored energy (density) of cold work can be considered as an alternative physical criterion. Later work (Rittel et al., 2008) identified a microstructural evolution, called dynamic recrystallization (DRX) as a local softening

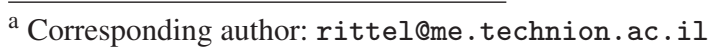

factor leading to the dynamic shear localization. The relative contribution of microstructural and local thermal evolutions was assessed by means of a numerical model by Osovski et al. (2013).

From a practical point of view, the concept of a critical strain energy density as a criterion for the initiation of the ASB, as well as a damage evolution criterion were illustrated by Dolinski et al. (2010), who demonstrated its feasibility by modeling 4 different laboratory experiments involving dynamic shear failure. Noam et al. (2014) extended this proof of feasibility by investigating the potential of the criterion to undergo scaling, and showed a simple penetration example based on the experimental work of Børvik et al. (1999).

One commonly used material that undergoes ASB is Rolled Homogeneous Armor (RHA), as defined in the military standard: MIL-DTL-12560J (MR). This steel is widely used in the assessment of the ballistic performance of various ammunitions. Shear bands in RHA have been reported in various works from microstructural and mechanical standpoints (Bassim et al. (2013); Fellows and Harding (2001); Gray et al. (1994)).

As of today, the emerging picture is that reliable prediction, through numerical modeling, of ballistic failure in general is still in its infancy. Putting aside extremely complex numerical models which are not implemented by the engineering community, one can find several (over)

This is an Open Access article distributed under the terms of the Creative Commons Attribution License 4.0, which permits unrestricted use, distribution, and reproduction in any medium, provided the original work is properly cited. 
simplified simulations, generally of thin plates, which are not always supported by experimental work, not to mention purely numerical works whose validity has not been verified.

What would be desirable is a simple (as much as can be) approach to model ballistic failure in a realistic way on the one hand, to an extent where the simulations can also have a predicting power. On the other hand, it is the authors' opinion that such an approach should be based on iterative numerical-experimental work, consisting of the following phases. The first phase is the calibration of the failure model, criteria and parameters based on one or more experiments, once a satisfactory convergence of the solution has been defined. The next phase, validation of the above-mentioned choices, consists of successfully replicating additional experimental tests results without changing any numerical parameter. Once this validation phase has been established, it can be concluded that the numerical tool has a predictive capability for the selected set of materials (target-projectile). Moreover, once validated, such an approach can be extended in a straightforward manner to additional materials systems.

The above paragraph is precisely the goal of the present paper. We investigate by means of carefully controlled ballistic impact experiments, the response of thick RHA steel. The chosen projectile is $\varnothing 20[\mathrm{~mm}]$ fragment simulating projectile (FSP), a standard and commonly used projectile. Furthermore, the classical failure criterion based on a critical strain is evaluated vs. the energy density criterion, and shown to be inadequate for the purposes of this research.

\section{Energy failure criterion and its implementation}

The failure criterion consists of a critical level of the plastic strain energy density $W_{\text {crit }}$ reached in each element, given by:

$$
W_{c r i t}=\int_{0}^{\alpha} S_{i j} d \varepsilon_{i j}^{P}=\int_{0}^{\alpha} \sigma_{M} d \varepsilon_{e q}^{P} .
$$

Where $S_{i j}$ and $\varepsilon_{e q}^{P}$ are the deviatoric stress and plastic strain tensor components, $\sigma_{M}$ and $\varepsilon_{e q}^{P}$ are the Mises stress and equivalent plastic strain. The upper integration limit $\alpha$ is selected as the level of strain at which the structural strength starts to deteriorate, $\tilde{\varepsilon}_{e q}^{\text {crit }}$. From this point on, the element starts to fail gradually, rather than abruptly, according to:

$$
\tilde{\sigma}_{e q}=\tilde{\sigma}_{e q}^{*}\left(1-D^{b}\right)
$$

Where $\tilde{\sigma}_{e q}^{*}$ is the current equivalent stress level at a given equivalent strain $\tilde{\varepsilon}_{e q} \geq \tilde{\varepsilon}_{e q}^{\text {crit }}, D$ is the level of damage in the element and $b$ is a constant. In the present work the parameter $b=1$, therefore only one adjustable parameter is needed for calibration, i.e. $W_{f r a c}$ as mentioned in the formulation below. $W_{\text {frac }}$ is the plastic strain energy density when the stress drops to zero.

The damage evolution is given by:

$$
D=\left\{\begin{array}{cl}
0 & W \leq W_{\text {crit }} \\
\frac{W-W_{\text {crit }}}{W_{\text {rac }}-W_{\text {crit }}} & W \geq W_{\text {crit }}
\end{array}\right\}
$$

This criterion was implemented via a user-subroutine (VUSDFLD) in the commercial finite element code Abaqus explicit V6.12 (2012), as presented in the appendix of Dolinski et al. (2010).

Another important point is the strength decay, damage evolution, after the critical energy density is met. The decay is exponential due to the formulation of the damage. Note that $W=\int_{0}^{\alpha} \sigma_{M} d \varepsilon_{e q}^{P}$, and although the material's strength might be constant in some cases like the steel of the current work, when $D>0 D>0$, the strength decreases causing a smaller $d W$ for each increment $d \varepsilon_{e q}^{P}$.

\section{Experimental setup}

The selected experimental setup was dictated by controllability, reproducibility, and selection of commonly used armor steel that fails by adiabatic shear. Moreover, we performed the experiments in a way that their simulation would be as simple as possible, meaning, axisymmetric simulation with simple homogeneous and isotropic materials.

Projectile: A standard 20[mm] FSP (Fragment Simulating Projectile) was used. In order to simplify the simulation, the "nose" of the projectile was trimmed prior the experiment, such as to obtain an axisymmetric problem. The FSP is made of 4340 steel, with a hardness of $\mathrm{R}_{C}=30$. The constitutive equation for this material was chosen as Johnson Cook equation, and the parameters were taken from their original article (Johnson and Cook, 1983). Note that this material is very ductile, so that damage and fracture of the projectile are not taken into account in the simulations.

Target: The experimental target was a $25 \pm 0.1[\mathrm{~mm}]$ thick RHA (Rolled Homogeneous Armor) plate, with a hardness of $\mathrm{R}_{\mathrm{C}}=39$.

\section{Experimental results}

A total of 9 shots were fired towards two different target plates. Three shots were fired at the first plate (Fig. 1a), and the remaining 6 shots stroke the second plate, as shown in Fig. 1b. All the relevant experimental parameters are listed in Table 1 . The range of velocities spanned from $1150[\mathrm{~m} / \mathrm{s}]$ to $1920[\mathrm{~m} / \mathrm{s}]$.

At velocities of $1322[\mathrm{~m} / \mathrm{s}]$ and below, the plate was penetrated but not perforated. The depth of penetration (DOP) depends of course on the impact velocity. It is interesting to note that, even at quite low impact velocity $(1248[\mathrm{~m} / \mathrm{s}])$, the rear of the plate contains a crack, which may remind of brittle fracture. This fracture is due to the tensile waves that are reflected from the free surface of the plate's back.

Another interesting result occurred when the plate was hit at a velocity of $1394[\mathrm{~m} / \mathrm{s}]$ (plate \#2). This is the minimal velocity at which the plate was totally perforated in the experiments. The plate's failure mechanism was undoubtedly by adiabatic shear banding. First of all, the fracture surface is observed to undergo extensive plastic deformation, for which a plug was formed and recovered. 
Table 1. Summary of the experimental results. "P" indicates full perforation.

\begin{tabular}{|c|c|c|c|c|c|c|}
\hline $\begin{array}{c}\text { Shot } \\
\text { number }\end{array}$ & $\begin{array}{c}\text { Measured } \\
\text { impact } \\
\text { velocity } \\
\mathbf{\pm 2 0}[\mathbf{m} / \mathbf{s}]\end{array}$ & $\begin{array}{c}\text { Depth of } \\
\text { penetration or } \mathbf{P}) \\
\text { perforation } \\
\mathbf{\pm 0 . 0 5}[\mathbf{m m}]\end{array}$ & $\begin{array}{c}\mathbf{F S P} \\
\text { weight } \\
\mathbf{\pm 0 . 0 1}[\mathbf{g r}]\end{array}$ & $\begin{array}{c}\text { FSP } \\
\text { length } \\
\mathbf{\pm 0 . 0 2}[\mathbf{m m}]\end{array}$ & $\begin{array}{c}\text { Inner } \\
\text { crater } \\
\text { diameter } \\
\mathbf{\pm 0 . 0 5}[\mathbf{m m}]\end{array}$ & $\begin{array}{c}\text { Outer } \\
\text { crater } \\
\text { diameter } \\
\mathbf{\pm 0 . 0 5}[\mathbf{m m}]\end{array}$ \\
\hline $\mathbf{1}$ & 1920 & $\mathrm{P}$ & 46.74 & 20.68 & $49.20-54.05$ & $28.95-30.45$ \\
\hline $\mathbf{2}$ & 1715 & $\mathrm{P}$ & 47.68 & 21.06 & $48.35-49.60$ & $27.15-28.90$ \\
\hline $\mathbf{3}$ & 1460 & $\mathrm{P}$ & 46.60 & 20.95 & $41.70-42.50$ & $23.60-26.70$ \\
\hline $\mathbf{4}$ & 1466 & $\mathrm{P}$ & 46.36 & 20.5 & $41.05-43.90$ & $22.65-24.75$ \\
\hline $\mathbf{5}$ & 1256 & 14.09 & 46.89 & 20.73 & $39.45-41.15$ & - \\
\hline $\mathbf{6}$ & 1248 & 13.74 & 46.75 & 20.66 & $38.45-39.10$ & - \\
\hline $\mathbf{7}$ & 1322 & 16.8 & 45.94 & 20.0 & $39.45-40.70$ & - \\
\hline $\mathbf{8}$ & 1394 & $\mathrm{P}$ & 46.51 & 20.58 & $39.85-41.70$ & $21.35-26.90$ \\
\hline $\mathbf{9}$ & 1151 & 11.05 & 46.79 & 20.32 & $36.30-37.40$ & - \\
\hline
\end{tabular}
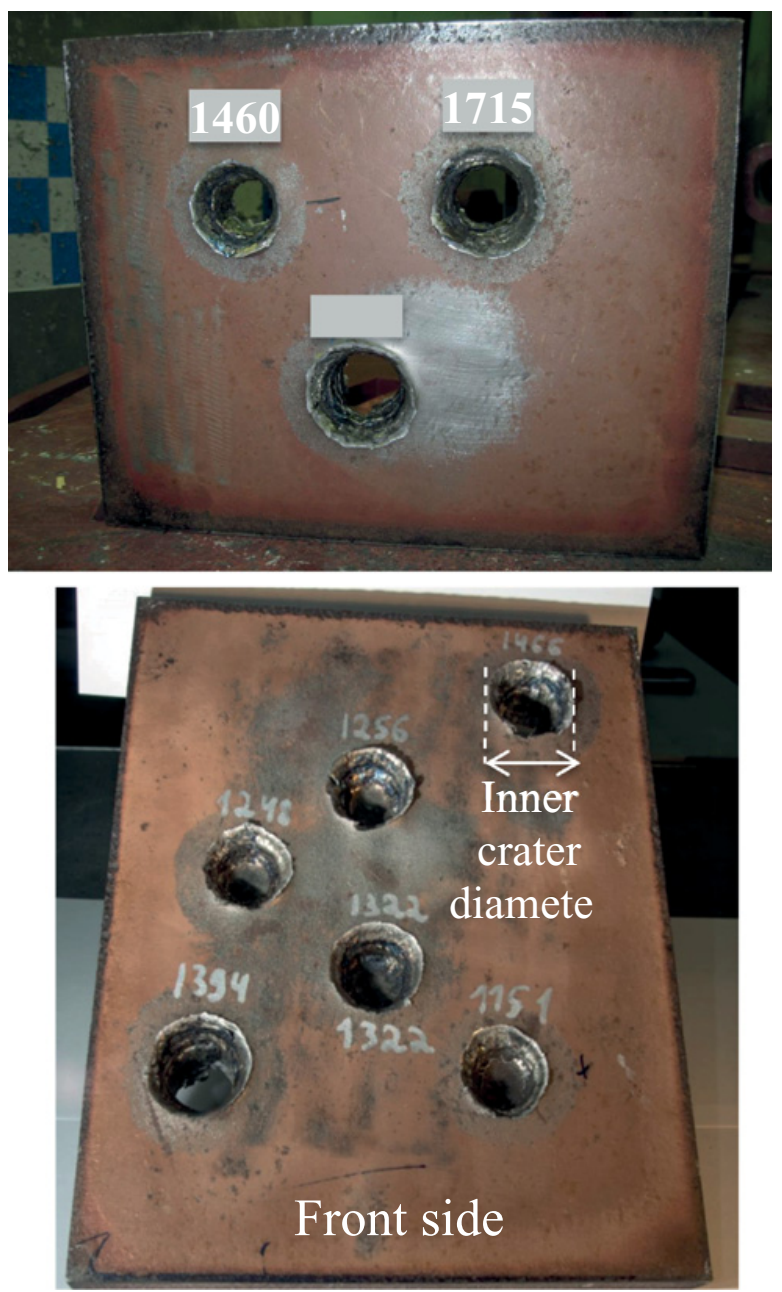

Figure 1. Plate \#1 (upper) and plate \#2 (lower). The numbers near the holes indicate the impact velocity in $[\mathrm{m} / \mathrm{s}]$.

Table 1 summarizes the experimental results, including the impact velocity, FSP parameters, perforation or DOP and crater diameters.

It must be noted that the results are not "perfect", meaning, the craters are not circular but rather elliptic, with an axes ratio of up to 1.1 . Therefore the crater

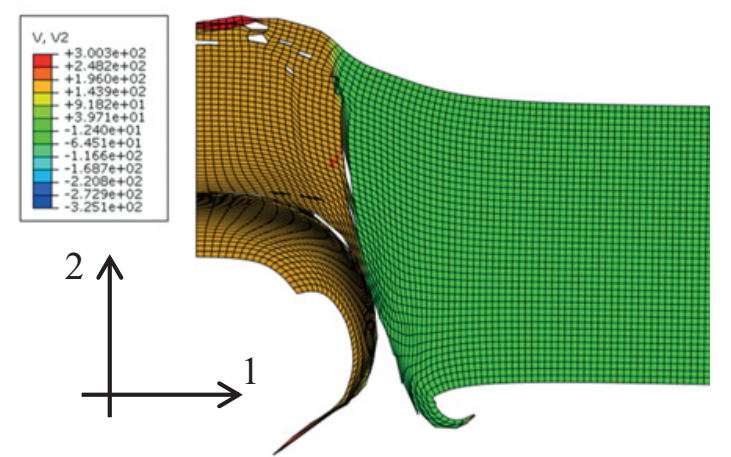

Figure 2. Velocity (V2) contour for impact velocity of $1400[\mathrm{~m} / \mathrm{s}]$ at $\mathrm{t}=42[\mu \mathrm{s}]$.

dimensions are given as a range of values in Table 1. Another noticeable scatter lies in the projectile dimensions and weight. The scatter in its length is about $\pm 2.5 \%$. A representative length of $20[\mathrm{~mm}]$ was chosen for all the simulated projectiles, as shown in the next section.

\section{Numerical results and comparison to experiments}

Impact velocity of $1400[\mathrm{~m} / \mathrm{s}]$ :

At this impact velocity, the plate was fully perforated by plugging, i.e. adiabatic shearing. Figure 2 shows the velocity contour along the impact axis. This picture is taken $42[-]$ after the impact. A fully developed plug is visible. The calculated plug dimensions and velocity are very similar to the experimental values, as summarized in Table 2. A fracture inside the plug causes elements to overlap, so that actual dimensions of the plug cannot be assessed. Moreover, the plug is not perfectly cylindrical but rather conical, and the simulation does not include frictional contact between the plug and the crater, as mentioned earlier regarding interaction between bodies.

Yet, the same energetic failure criterion is able to reproduce the plugging process with a high resemblance (qualitative and quantitative) to the experimental results. Very sensitive experimental results, such as the plug exit velocity, are captured with a relative error of less than $10 \%$. 
Table 2. Comparison of experimental and numerical results for impact velocity of $1400[\mathrm{~m} / \mathrm{s}]$.

\begin{tabular}{|l|l|l|}
\hline & Experiment & Simulation \\
\hline Impact velocity & $1394 \pm 20[\mathrm{~m} / \mathrm{s}]$ & $1400[\mathrm{~m} / \mathrm{s}]$ \\
\hline Crater maximal & $39.45-40.7 \pm 0.1[\mathrm{~mm}]$ & $39.53[\mathrm{~mm}]$ \\
\hline diameter & & \\
\hline Plug exit velocity & $196 \pm 20[\mathrm{~m} / \mathrm{s}]$ & $180 \pm 20[\mathrm{~m} / \mathrm{s}]$ \\
\hline Plug diameter & $20.9-26.2 \pm 0.1[\mathrm{~mm}]$ & $26.05[\mathrm{~mm}]$ \\
\hline Plug height & $22.2 \pm 0.1[\mathrm{~mm}]$ & $20.1[\mathrm{~mm}]$ \\
\hline
\end{tabular}

\section{Discussion and conclusions}

This paper has presented an alternative approach to the treatment of dynamic shear localization to the well accepted procedures based on a critical strain to failure.

We use here a simple, yet physical, criterion based on the plastic strain energy density for the onset of shear localization. A preliminary validation of the criterion can be found in Dolinski et al. (2010) who analyzed relatively simple examples of experiments involving dynamic shear localization.

The case studied in this paper is far more complex and therefore rather challenging. In this work, we have presented a careful series of experiments in which, all parameters being identical, the impact velocity of a standard projectile is carefully controlled.

The physical failure mechanisms are well defined and consist of adiabatic shear and spalling (tensile). For each experiment, a different final state of the impacted plate differs, ranging from partial penetration, through penetration and full penetration with fragmentation, according to the impact velocity.

The goal of this study was to demonstrate that those three kinds of final states can be successfully modeled using the same approach and a fixed set of parameters.

The first issue to be addressed is that of the pathological mesh sensitivity of dynamic shear localization problems. While several sophisticated methods have been devised, they are often only partly supported by experimental evidence, and almost always quite complex to include in a finite element code. Consequently, an engineering approach was adopted here, namely an iterative determination of the mesh parameters and material properties that would yield an accurate replication of all the measurable experimental parameters, mostly of a geometrical nature. This was achieved based on a preliminary set of experiments. Once a satisfactory prediction was reached, all the subsequent experiments were modeled with this exact same set of parameters without further adjustment. The outcome was quite satisfactory, and validated the selected approach.
It is therefore suggested that a similar approach be adopted to model additional penetration/perforation problems for other sets of target materials and projectiles.

Another outcome of this work was a re-assessment of a critical strain to failure as the dynamic shear failure criterion. Indeed, when the above-mentioned approach was applied using this calibrated parameter on one set of experiments, it was found that the other experiments could not be faithfully replicated unless the failure strain is readjusted, which is not physical.

A comparison of the dynamic strain energy density and the damage evolution model with the critical strain criterion revealed that the underlying reason for the inadequacy of the latter and the adequacy of the former lies in the evolution of the damage. Namely, it was found that the stress decrease is linear in strain for the strain criterion, whereas with the energy criterion, strength decreases exponentially, as shown in Fig. 1. One should note that this exponential decay not only leads to satisfactory numerical results, but it also matches the experimental observations of .()Fellows and Harding (2001) who investigated RHA steel in the same procedure of Marchand and Duffy (1988).

Finally, to highlight the specific nature of the present work, one can note that:

- The present work is not an experimental study with a large statistical database. It nevertheless carefully emphasizes specific impact velocities for which specific failure mechanisms operate.

- The same criterion predicts the initiation and dictates the propagation of the ASB.

- The overall approach exposed here is relatively simple and physical, with a minimum number of adjustable parameters.

- This ASB failure criterion is not rate dependent, as shown experimentally by Osovski et al. (2012).

The following conclusions can be now drawn from this work:

- Ballistic penetration/perforation can be successfully modeled using a simple plastic strain energy density and damage model with one adjustable parameter.

- This study extends and validates further the proposed criterion.

- A detailed combined experimental-numerical approach is proposed to calibrate the parameters of the numerical model.

- The concept of a critical strain to failure is shown to be inadequate for the purpose of numerically simulating and predicting ballistic failure.

- All in all, the proposed approach is relatively simple and applicable, and should therefore be of practical interest to the engineering community. 